\section{BMJ Open} Ophthalmology

\title{
Correlation of features on OCT with visual acuity and Gass lesion type in Best vitelliform macular dystrophy
}

\author{
Razek G Coussa, ${ }^{1,2}$ Christopher R Fortenbach, ${ }^{1,2}$ D Brice Critser, ${ }^{1,2}$ \\ Malia M Collins, ${ }^{1,2}$ Budd A Tucker, ${ }^{1,2}$ Robert F Mullins, ${ }^{1,2}$ Elliott H Sohn, ${ }^{1,2}$ \\ Edwin M Stone, ${ }^{1,2}$ Ian C Han (1) ${ }^{1,2}$
}

To cite: Coussa RG, Fortenbach CR, Critser DB, et al. Correlation of features on OCT with visual acuity and Gass lesion type in Best vitelliform macular dystrophy. BMJ Open Ophthalmology 2021;6:e000860. doi:10.1136/ bmjophth-2021-000860

- Additional supplemental material is published online only. To view, please visit the journal online (http://dx.doi. org/10.1136/bmjophth-2021000860).

The key findings from this study were presented at the Annual Meeting of the Macula Society, February 2021.

Received 20 July 2021 Accepted 21 November 2021

\section{ABSTRACT}

Objective To correlate structural features seen on optical coherence tomography (OCT) with best-corrected visual acuity (BCVA) and Gass lesion type in patients with Best vitelliform macular dystrophy (BVMD).

Methods and analysis This is a retrospective case series of consecutive patients with molecularly confirmed BEST1-associated BVMD. OCT scans were reviewed for lesion status and presence of subretinal pillar, focal choroidal excavation (FCE), intraretinal fluid or atrophy. Available OCT angiography images were used to evaluate for the presence of choroidal neovascularisation (CNV). These features were then correlated with BCVA and Gass lesion type.

Results 95 eyes from 48 patients (mean age 38.9 years, range 4-87) were included. The presence of a pillar (24.2\%), FCE (20.0\%) and atrophy (7.4\%) were associated with poor BCVA $(p<0.05)$. Gass lesion type 1 eyes were correlated with good BCVA (LogMAR <0.4) whereas type 5 eyes had poor BCVA (LogMAR >0.4). Among 65 eyes with longitudinal data (mean follow-up 5.1 years), 7 eyes $(10.8 \%)$ reverted from higher to lower Gass lesion type; of these, 4 eyes (57.1\%) had CNV responsive to intravitreal anti-vascular endothelial growth factor treatment.

Conclusion OCT-based structural features are readily identifiable in patients with BVMD and have prognostic importance due to their correlation with BCVA.

\section{INTRODUCTION}

Check for updates

(C) Author(s) (or their employer(s)) 2021. Re-use permitted under CC BY-NC. No commercial re-use. See rights and permissions. Published by BMJ.

${ }^{1}$ Department of Ophthalmology and Visual Sciences, University of lowa, lowa City, lowa, USA ${ }^{2}$ Institute for Vision Research, University of lowa, lowa City, Iowa, USA

\section{Correspondence to} Dr lan C Han; ianhanmd@gmail. com
Best vitelliform macular dystrophy (BVMD) is one of the most common macular dystrophies, with an estimated frequency of about 1 in $70000 .{ }^{12}$ BVMD results from pathologic variants in BEST1, ${ }^{1}$ which encodes bestrophin-1, an integral transmembrane protein located on the basolateral aspect of retinal pigment epithelial (RPE) cells. $^{3-5}$ The age at which vitelliform lesions first become detectable with ophthalmoscopy in individuals affected with BVMD ranges from as early as the first year of life to beyond the seventh decade. ${ }^{6}$ The subretinal material within vitelliform lesions can adopt various anatomical configurations during the lifetime of the patient, which Gass interpreted to be a series of stages:

\section{Key messages}

What is already known about this subject?
Vitelliform lesions in patients with Best disease can
progress over time through one or multiple stages
(Gass lesion types).
What are the new findings?
Structural features on optical coherence tomography
(OCT) are easily identifiable, with better intergrader
agreement and stronger correlation with visual acu-
ity than traditional Gass staging. Vitelliform lesions
can reverse from higher to lower Gass lesion type
over time, and treatment of choroidal neovascular-
isation (CNV) may play a role.
How might these results change the focus of
research or clinical practice?
Clinicians should be aware of OCT-based features in
patients with Best disease that have close correla-
tion with visual acuity. Future studies may focus on
the role of CNV in the evolution of vitelliform lesions
over time.

pre-vitelliform (stage 1), vitelliform (stage 2), pseudohypopyon (stage 3), vitelleruptive (stage 4) and atrophy and/or fibrosis (stage 5). ${ }^{78}$ However, Gass' classification was introduced in 1974, well before the advent of optical coherence tomography (OCT), ${ }^{9}$ and not surprisingly, there are often discrepancies between Gass categories and OCT features. ${ }^{10}$ Because not all patients progress steadily and sequentially through the ophthalmoscopic configurations described by Gass, we prefer the term 'lesion types' rather than 'stage'. 112

To date, few studies have characterised the natural history of BVMD with OCT imaging, and in particular, the pathophysiologic mechanisms responsible for the transition from vitelliform to atrophic lesions are unknown. Choroidal neovascularisation (CNV) is typically thought to occur late in the disease (sometimes considered Gass stage 6), ${ }^{13-17}$ but whether CNV is a sequelum of atrophy 
or actually contributes to collapse of vitelliform lesions remains a matter of debate. Parodi and colleagues recently used OCT angiography (OCTA) to demonstrate that many patients with BVMD possess subclinical CNV, suggesting that $\mathrm{CNV}$ likely arises earlier in the disease process and may play an important role in the evolution of vitelliform lesions over time. ${ }^{18}$ However, longitudinal data with OCTA, a relatively nascent imaging modality, are currently limited; we thus sought to pursue insights into this process using OCT, a modality for which we have years of follow-up imaging available in patients with BVMD.

In this study, we use longitudinal OCT to evaluate the prevalence of structural OCT features and their evolution over time in a large cohort of patients with BVMD. We further correlate these OCT-based features with visual acuity, Gass lesion types and clinical diagnosis of CNV. The findings highlight OCT-based features that are readily identifiable, that possess clinical utility in their correlation to visual acuity and that provide insight into pathophysiologic mechanisms.

\section{MATERIALS AND METHODS}

This was a retrospective chart review of consecutive patients with molecularly-confirmed BEST1-related BVMD seen at the University of Iowa Hospitals and Clinics between 2008 and 2020. Institutional review board approval was obtained from the University of Iowa Hospital and Clinics. The research adhered to the tenets of the Declaration of Helsinki and was conducted in accordance with regulations set forth by the Health Insurance Portability and Accountability Act. Demographic data including age at each visit, gender and genotype were recorded. Patients or the public were not involved in the design, or conduct, or reporting, or dissemination plans of our research. Patients with BVMD with comorbid ophthalmic conditions affecting adequate image acquisition (eg, corneal pathology) or comorbid retinal disease (eg, diabetic retinopathy, age-related macular degeneration (AMD)) were excluded. The Snellen best-corrected visual acuity (BCVA) for each eye and each visit was recorded and converted to its LogMAR equivalent, as previously described. ${ }^{19}$ Patients with a clinical diagnosis of CNV were identified, and any treatment (eg, with intravitreal anti-vascular endothelial growth factor (antiVEGF) therapy) was recorded.

Multimodal imaging including colour fundus photography and spectral domain OCT (Spectralis OCT; Heidelberg Engineering, Heidelberg, Germany) from each patient with BVMD at each visit were included for analysis. OCT scan protocols included fovea-centred macular volume scan protocols that ranged from $20^{\circ} \times 20^{\circ}$ to $30^{\circ} \times 20^{\circ}$, with 512,768 or 1024 (horizontal) $\times 496$ (vertical) pixel density, and had 19, 25 or 49 B-scans. All longitudinal scans were acquired using registration to prior scans. The segmentation for each scan was reviewed for algorithm errors (RC), and no manual correction was needed for centre subfield retinal thickness measurements, which were recorded for each eye and visit.

Each OCT volume scan was evaluated for the status of the vitelliform lesion as either (1) pre-vitelliform (subfoveal thickening of the cone outer segment tips (COST) line without vitelliform deposit or subretinal fluid (SRF)) (online supplemental figure 1A,B), (2) solid vitelliform (dome-shaped homogeneously reflective lesion without overlying fluid) (online supplemental figure 1C,D) or (3) vitelliform neurosensory detachment (hyporeflective space between the photoreceptors and RPE) (online supplemental figure 1E-H). Other OCT features analysed included presence of (4) subretinal pillar, ${ }^{20}$ defined as a sharply angled $\left(>45^{\circ}\right.$ relative to Bruch's membrane on OCT line scan, which has a 3:1 axial stretch) focal elevation of the RPE with shadowing to the choroid (online supplemental figure $1 \mathrm{G}-\mathrm{H}),{ }^{21}{ }^{22}$ (5) focal choroidal excavation (FCE) (online supplemental figure 1I,J), ${ }^{23-25}$ (6) intraretinal fluid (IRF) (online supplemental figure $1 \mathrm{~K}-\mathrm{L}$ ) or (7) atrophy and/or fibrosis, defined as the loss of outer retinal layers including the ellipsoid zone band, COST or external limiting membrane (online supplemental figure 1M-P). For eyes with FCE, the subtype (conforming vs non-conforming) was also determined based on prior definitions for these subtypes. ${ }^{2326} 27$ The presence or absence of these features was determined by two independent ophthalmologist graders (RC, CRF), with an additional grader (IH), serving to arbitrate any disagreement. Gass lesion type was determined using fundus photography and OCT imaging, including corresponding en face near infrared reflectance images.

When available, spectral domain OCTA imaging (Cirrus HD-OCT 5000 with Angioplex, Zeiss Meditec, Dublin, California, USA) was also evaluated for the presence of visible $\mathrm{CNV}$ (defined as an identifiable vascular network above Bruch's membrane, with visible doppler flow on line scans through the lesion) in the outer retina to choriocapillaris (ORCC) slab by two independent graders (RC, IH). OCTA scan protocols included 3 $\mathrm{mm} \times 3 \mathrm{~mm}$ and $6 \mathrm{~mm} \times 6 \mathrm{~mm}$ scans centred on the fovea.

\section{Cross-sectional analysis}

For each eye and graded OCT feature, we calculated the number and percentage of eyes with that feature, as well as the mean, median and range of the LogMAR BCVA measured at each corresponding visit. We then used paired t-tests to compare the overall BCVA and age of eyes with a given feature to those without that feature. A Kruskal-Wallis $\mathrm{H}$ test and post hoc Mann-Whitney $\mathrm{U}$ test analyses were conducted to investigate differences in the prevalence of OCT features by age group. Pearson correlations were used to investigate the relationship between the OCT features at baseline. To determine whether any of the examined OCT features at the initial visit were associated with or predictive of a BCVA equal to or worse than 0.4 LogMAR (20/50 Snellen equivalent), a univariate logistic regression analysis was conducted. The visual acuity cut-off was determined prior to analysis 
based on a previous study we performed (178 eyes from 89 patients) that suggested 0.4 LogMAR as an inflection point for decline in visual acuity (online supplemental figure 2). ${ }^{28}$ We also carried out a series of t-tests and Mann-Whitney U tests to investigate: (1) the differences in BCVA, central subfield thickness (CST) and age among eyes with the characteristic OCT features and those without; and (2) OCT features among eyes with BCVA better or worse than 0.4 LogMAR.

\section{Longitudinal analysis}

For each eye with available follow-up data at least 9 months apart, the same OCT features noted above were recorded as described above. For each feature, we used Mann-Whitney U test to compare the differences in prevalence and respective BCVA between the initial and final visits. We also carried out a series of Mann-Whitney U tests to investigate the differences in OCT features at the last visit among eyes with $\mathrm{CNV}$ and those without CNV.

\section{Statistical analysis}

Statistical analyses were performed using Microsoft Excel (Microsoft Corporation, Redmond, Washington, USA) and IBM SPSS Statistics, V.26 (IBM Corporation, Armonk, New York, USA). Statistical significance was set at $\mathrm{p}<0.05$ for all comparisons.

\section{RESULTS}

A total of 95 eyes from 48 patients with BVMD were included for analysis. The mean age was 38.9 years (median: 36.0; range: 4-87) and the majority of the patients were men $(60.4 \%, 29 / 48)$. The mean LogMAR BCVA was 0.31 (median LogMAR: 0.30; range: -0.12 to 1.00). The mean CST on OCT was $346.7 \mu \mathrm{m}$ (median: 322; range: 73-627). Overall grader agreement was excellent $(90 \%)$ for all OCT features, with the lowest agreement $(83 \%)$ associated with the presence of a pillar, and highest agreement associated with the presence of a pre-vitelliform lesion or SRF (96\% for both features). However, overall agreement between graders was substantially lower for Gass lesion types (46\%), and the agreement was lower at follow-up compared with baseline (38\% vs 53\%, respectively; online supplemental table 1).

\section{Cross-sectional analysis}

The prevalence of specific OCT features at baseline, with corresponding BCVA and CST measurements, is shown in table 1. On initial evaluation, a vitelliform lesion with SRF was the most common feature $(61.1 \%)$, followed by subretinal pillar $(24.2 \%)$ and FCE $(20 \%)$. The majority of FCEs were non-conforming (17/19 eyes; 89.5\%). Atrophy $(7.4 \%)$ and a clinical diagnosis of $\mathrm{CNV}(10.5 \%)$ were less common. On univariate analysis, features associated with poor LogMAR VA included pillars, FCE, IRF and atrophy, and LogMAR BCVA of eyes with each of these features was worse when compared with eyes without these features $(p<0.05$ for all comparisons, table 1$)$. None of the features were found to reach statistical significance on multivariate analysis. When evaluating age, the only comparison that reached statistical significance was eyes possessing pillars compared with those without $(\mathrm{p}=0.04$, table 1).

\section{Stratification by BCVA better or worse than 20/50 (LogMAR \\ 0.4)}

When stratifying the groups for analysis based on baseline BCVA, there were 60 eyes $(63.2 \%)$ with good vision (BCVA $\geq 0.4 \operatorname{LogMAR}$ ). The mean age of these patients was 37.5 years (median: 34.0 ; range: $4-87$ ), and mean CST was $335.7 \mu \mathrm{m}$ (median: 334; range: $210-570$ ). The remaining 35 eyes $(36.8 \%)$ had poor vision $(\mathrm{BCVA}<0.4$ LogMAR). The mean age of the poor vision group was 41.2 years (median: 45.0; range: 7-84 years) and mean CST was 331.3 $\mu \mathrm{m}$ (median: 300; range: 73-627). Patients with poor vision were older and had thinner retinas than those with good vision, but these differences were not statistically significant. Eyes with good vision had a higher rate of pre-vitelliform and solid vitelliform lesions compared with those with poor vision (table 2). Eyes with poor vision had a higher rate of pillar, FCE, IRF, CNV as well as atrophy than those with good vision (table 2). Eyes with poor vision had a significantly higher proportion of eyes graded as Gass type 5 and lower proportion as stage $1(\mathrm{p}<0.05)$. There was no statistically significant correlation between BCVA across Gass lesion types, and pairwise comparison of eyes with good and poor BCVA showed no statistically significant differences for eyes categorised as Gass lesion types 2, 3 or 4 .

\section{Longitudinal analysis}

OCT volume scans from 65 eyes from 33 patients were included in the longitudinal analysis. At baseline, mean age was 40.1 years (median: 42; range: $4-84$ ), and the majority of the patients were men $(60.6 \%, 20 / 33)$. Mean LogMAR BCVA was 0.34 (median: 0.3; range: -0.12 to 1) (online supplemental table 2). The mean follow-up period of 5.1 years (range $0.75-11$ years). Mean LogMAR BCVA (mean 0.27; median LogMAR 0.2; range -0.52 to 0.9 ) and CST (mean $340 \mu \mathrm{m}$; median 321; range 142-629) were lower than at baseline (online supplemental table 2).

Eyes generally progressed through more advanced Gass lesion types, and the prevalence of FCE, atrophy and CNV increased over time (online supplemental table 2). For eyes with FCE, the majority were the non-conforming subtype (15/17 eyes; $88.2 \%$ ). However, $10.8 \%$ ( $n=7$ eyes) exhibited a lower Gass lesion type on follow-up evaluation relative to baseline. Among these, four eyes (57.1\%) had CNV, and the lower Gass lesion type was observed following intravitreal anti-VEGF treatment. Ten eyes from five patients had available longitudinal OCTA imaging, and of these, $80 \%$ ( 8 out of 10 eyes from 4 patients) had a visible CNV on OCTA imaging. Representative cases illustrating the evolution of various OCT features over time are shown in figures 1-3 and described in detail below. 


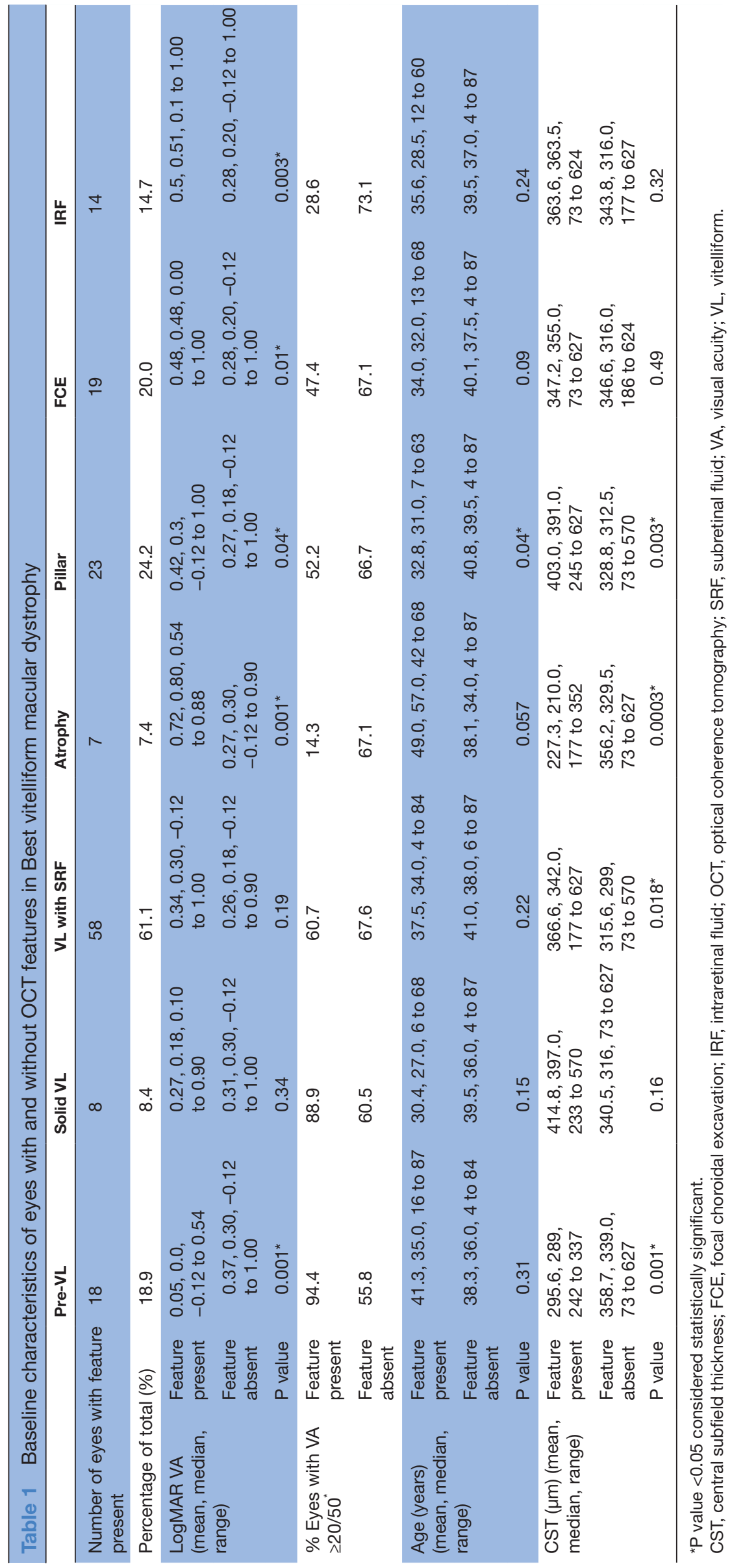

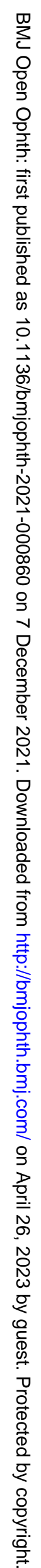


Table 2 Prevalence of eyes with specific optical coherence tomography (OCT)-based biomarkers and Gass lesion types stratified by best-corrected visual acuity better or worse than 0.4 LogMAR

\begin{tabular}{|c|c|c|c|}
\hline & BCVA <0.4 LogMAR $(\geq 20 / 50)$ & BCVA $\geq 0.4$ LogMAR $(<20 / 50)$ & $P$ value \\
\hline Number of eyes & 60 & 35 & - \\
\hline Age (Years) (mean, median, range) & $37.5,34,4$ to 87 & $41.2,45,7$ to 84 & 0.21 \\
\hline CST $(\mu \mathrm{m})$ (mean, median, range) & $335.7,334,210$ to 570 & $331.3,300,73$ to 627 & 0.15 \\
\hline Pre-VL (\%) & 28.3 & 2.9 & $0.02^{*}$ \\
\hline Solid VL (\%) & 13.3 & 2.9 & 0.2 \\
\hline SRF (\%) & 61.7 & 68.6 & 0.29 \\
\hline Pillar (\%) & 20 & 31.4 & 0.18 \\
\hline FCE (\%) & 15 & 28.6 & 0.14 \\
\hline $\operatorname{IRF}(\%)$ & 6.7 & 28.6 & $0.04^{*}$ \\
\hline Fibrosis and/or atrophy (\%) & 1.7 & 17.1 & 0.21 \\
\hline CNV (\%) & 6.7 & 17.1 & 0.2 \\
\hline \multicolumn{4}{|l|}{ Gass lesion type (\%) } \\
\hline 1 & 30 & 2.8 & $0.02^{*}$ \\
\hline 2 & 26.7 & 17.1 & 0.44 \\
\hline 3 & 21.7 & 22.9 & 0.93 \\
\hline 4 & 16.6 & 22.9 & 0.62 \\
\hline 5 & 5 & 34.3 & $0.02^{*}$ \\
\hline
\end{tabular}

${ }^{*} \mathrm{P}$ value $<0.05$ (Mann-Whitney $U$ test) considered statistically significant.

BCVA, best-corrected visual acuity; CNV, choroidal neovascularisation; CST, central subfield thickness; FCE, focal choroidal excavation; IRF, intraretinal fluid; SRF, subretinal fluid; VL, vitelliform.

\section{Representative case examples}

Case 1

Typical progression through Gass lesion types, with collapse of vitelliform lesion and development of atrophy. A man in his 50s with BVMD (BEST1, Gln96Glu heterozygote) presented with 20/20 visual acuity in the left eye. Fundus examination demonstrated a partially-layered vitelliform lesion with SRF (Gass type 3, figure 1A,B). After 2 years of follow-up, vision declined to 20/60, with resorption of SRF (Gass type 4, figure 1C,D). By 6 years of follow-up, BCVA remained 20/60 with eccentric fixation, and there was central atrophy (Gass type 5 , figure 1E,F).

Case 2

Rebound of a vitelliform lesion with SRF following treatment of CNV with intravitreal anti-VEGF therapy. A man in his 20s with BVMD (BEST1, Tyr227Asn heterozygote) was found to be $20 / 30$ in the right eye with an FCE and typical vitelliform lesion (Gass type 2) with SRF on OCT (figure 2A-A'). Three years later, he developed new distortion, with corresponding resorption of the SRF (Gass type 4) on OCT and a visible CNV on OCTA (figure 2B-B'). Following three sequential intravitreal anti-VEGF (bevacizumab $1.25 \mathrm{mg} / 0.05 \mathrm{~mL}$ ) injections, there was reconstitution of SRF (Gass type 2) and regression of the $\mathrm{CNV}$ (figure $2 \mathrm{C}-\mathrm{C}^{\prime \prime}, \mathrm{D}-\mathrm{D}^{\prime \prime}$ and $\mathrm{E}-\mathrm{E}^{\prime \prime}$ ).

Case 3

A teenage man with BVMD (BEST1, Arg218His heterozygote) was found to have BCVA $20 / 70$ in the right eye at initial evaluation with a central fibrotic pillar and SRF (figure 3A,B). By 5 years of follow-up, there had been spontaneous collapse of the pillar, with 20/80 visual acuity (figure 3C,D). By 7 years of follow-up, visual acuity measured 20/50 eccentrically with central atrophy (figure 3E), flattening of the fibrotic pillar, development of a FCE and minimal remaining SRF (figure 3F).

\section{DISCUSSION}

Gass' classification system for BVMD, which was based on fundoscopic findings and developed decades prior to OCT, has been widely used to describe macular lesions in BVMD. However, despite its widespread adoption, the subjectivity of Gass staging makes the scheme difficult to employ in many cases. By contrast, OCT-based evaluation now enables a more detailed, cross-sectional view of structural biomarkers that are relatively easy to identify and may provide better prognostic information. In our study, grader agreement was excellent for OCT-based classification of macular lesions, and OCT-based categorisation was far more consistent than assigning Gass lesion type (90\% overall grader agreement for OCT features vs $46 \%$ for Gass lesion type). OCT-based features also provide information that correlates closely with visual acuity. Specifically, the presence of a fibrotic pillar, FCE, IRF, CNV and atrophy were all associated with worse vision. Consistent with prior studies showing relatively little difference in BCVA between intermediate Gass stages, ${ }^{7}$ in our study, there were no significant differences between 
eyes graded as Gass types 2-4. Given the subjective nature of Gass typing and the stronger correlation of OCT-based structural features with BCVA, OCT-based categorisation may have a role for prognosis in addition to, or in place of, traditional Gass staging. ${ }^{10}$

To date, few studies have evaluated longitudinal structural changes on OCT in patients with BVMD. Previous case reports have shown that patients may not progress sequentially through Gass stages, and some eyes may even paradoxically reverse from higher (more advanced) stages to lower (less advanced) stages, even in the absence of any treatment. ${ }^{11} 12$ In our study, we encountered several examples $(10.7 \%$ of eyes with longitudinal follow-up) where patients reverted from higher to lower Gass lesion types (eg, rebound of a vitelliform lesion after initial lesion collapse). Interestingly, $57.1 \%$ (4 eyes) had CNV responsive to intravitreal anti-VEGF treatment.
CNV is traditionally thought to be a late-stage manifestation of BVMD, ${ }^{13-17}$ but the detection of $\mathrm{CNV}$ is particularly challenging with fluorescein angiography (eg, pooling within vitelliform lesions often limits the identification of CNV-related leakage). The presence of SRF on OCT is a useful indicator of CNV in other conditions such as exudative AMD. However, in patients with BVMD, SRF is routinely seen within vitelliform lesions, and the presence of SRF is associated with better visual acuity whereas loss of SRF (ie, collapse of vitelliform lesion) is a poor prognostic sign. As such, the presence of SRF is not a reliable sign of CNV in BVMD, and other imaging-based biomarkers (eg, fibrotic pillar, FCE, IRF) may be more useful. The presence of FCE and IRF have been well-described as OCT-based markers for CNV in other conditions. ${ }^{25-27} 29$ Fibrotic pillars are a relatively distinct finding in patients with BVMD, and recently, 


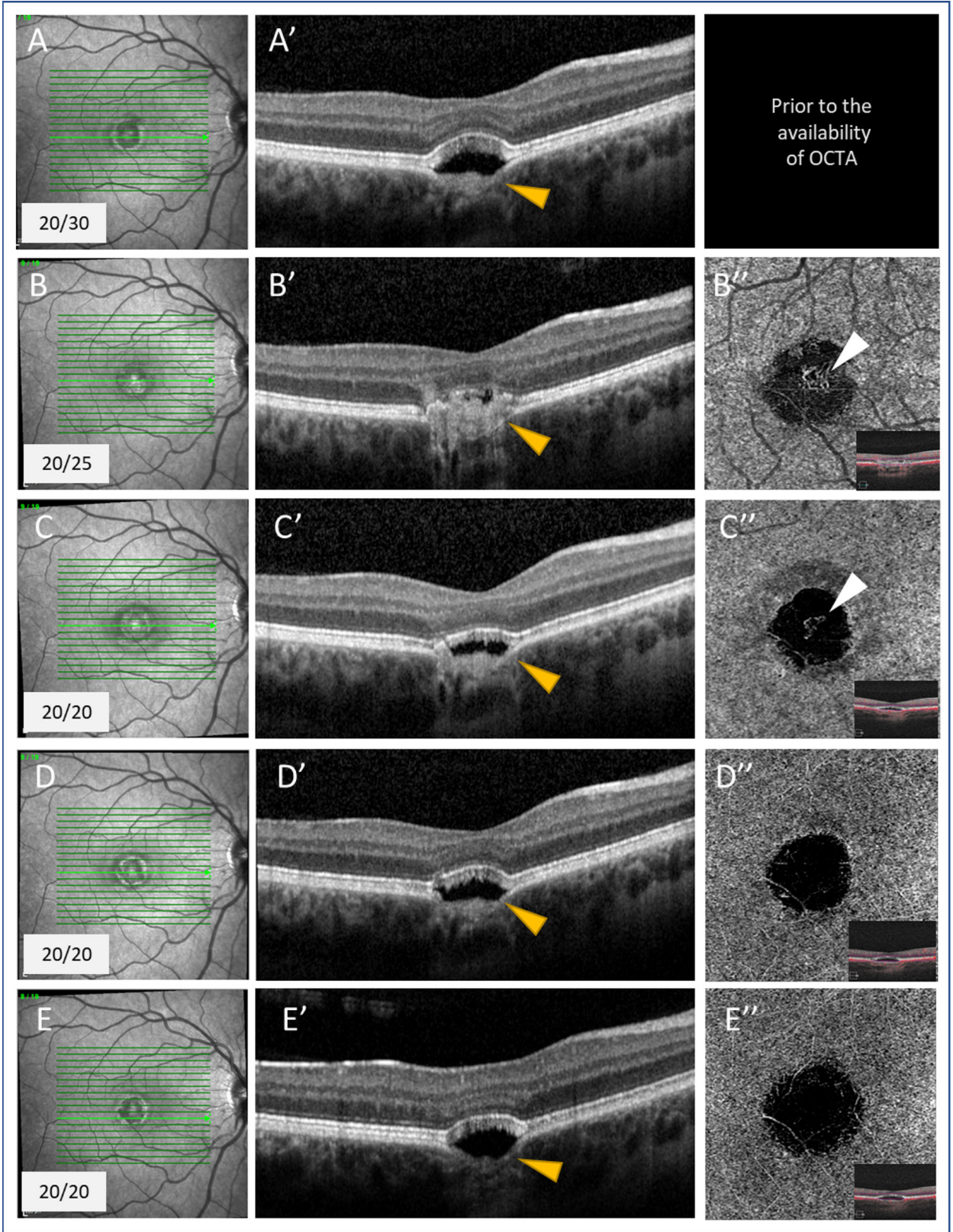

Figure 2 Case 2 illustrating collapse of vitelliform lesion due to choroidal neovascularisation (CNV) and rebound after antivascular endothelial growth factor (VEGF) treatment. A man in his 20s (BEST1, Tyr227Asn) was seen initially with bestcorrected visual acuity (BCVA) or 20/30 (A). Optical coherence tomography (OCT) showed a vitelliform lesion with subretinal fluid ( $A^{\prime}$; yellow arrow) as well as a non-confirming focal choroidal excavation. Three years later, he presented with new distortion and was found to have a BCVA of 20/25 with resorption of subretinal fluid and accumulation of hyperreflective material (B-B'; yellow arrow). Optical coherence tomography angiography (OCTA) of the outer retina to choriocapillaris (ORCC) showed a corresponding CNV (C", white arrow), and an intravitreal anti-VEGF treatment (bevacizumab $1.25 \mathrm{mg} / 0.05 \mathrm{~mL}$ ) was administered. One month after treatment, there was decreased distortion and improvement in BCVA to 20/20, with rebound of fluid and an incompletely regressed CNV on ORCC slab of the OCTA (C'), and a second intravitreal anti-VEGF injection was given. One month later, the vitelliform lesion had increased in height on OCT (D- $\left.D^{\prime}\right)$, with no visible CNV on OCTA ( $\left.D^{\prime \prime}\right)$. An additional anti-VEGF treatment was administered and follow-up a month later showed continued increase in height of the vitelliform lesion $\left(E-E^{\prime}\right)$ without visible remaining $C N V\left(E^{\prime \prime}\right)$.

OCTA has been used to demonstrate that these subretinal lesions often contain abnormal choroidal vascular networks. ${ }^{18}$ As shown in Case 3 (figure 3), these peaked subretinal lesions are highly-hyperreflective on OCT, and may collapse or involute, resulting in FCE, loss of SRF and decrease in visual acuity.
The relatively high prevalence of structural OCT features associated with $\mathrm{CNV}$ suggests that $\mathrm{CNV}$ may actually be an early rather than late-stage finding in patients with BVMD, and that $\mathrm{CNV}$ may play an important role in the transition from vitelliform lesions to atrophy. Individual examples such as Case 2 (figure 2) are thus 
particularly provocative and demonstrate that vitelliform lesions can rebound after initial collapse, violating the expected advancement through progressively higher Gass stages. As shown in this example case, the collapse of a vitelliform lesion was associated with the presence of FCE and CNV on OCT and OCTA. Treatment with anti-VEGF therapy resulted in regression of any visible CNV on OCTA, rebound of the vitelliform lesion with $\mathrm{SRF}$ and improvement in BCVA. These findings suggest that CNV may play an important role in the pathophysiology of BVMD, particularly pertaining to progression from earlier stages to vitelleruptive or atrophic lesion types. Early detection of the presence of CNV, including with newer imaging modalities such as OCTA, may allow for treatment that decreases the likelihood of vision loss. Studies with longitudinal OCTA-based are needed to further clarify the relationship between structural and vascular changes over time.

Our study had several limitations, including its retrospective nature and the lack of standardised volume scan protocols with regard to scan density or follow-up intervals. As such, we focused on qualitative OCT-based features that might be readily identifiable by a clinician. Our group has previously demonstrated that photoreceptor outer segment length varies in $\mathrm{BVMD},{ }^{20}$ including by genotype $^{30}$; future studies may use retinal sublayer thickness analyses to investigate quantitative differences by genotype. Due to the relative nascency of OCTA imaging, we had longitudinal OCTA in only a few patients, including those with high clinical suspicion for CNV. More work is needed to correlate structural features (eg, pillars, FCE) with the presence or absence of a visible CNV on OCTA, 
perhaps with swept-source OCTA given its improved ability to study choroidal details. Similarly, though prior OCTA-based studies have demonstrated choriocapillaris flow loss in patients with BVMD, ${ }^{18}$ the pathophysiologic sequence of choroidal flow loss, FCE formation and CNV remains unclear. Leveraging of data from multimodal imaging and long-term follow-up studies may pave the way for a more comprehensive staging system that parallels the pathophysiology of BVMD and gives clinicians a better sense of prognosis.

Correction notice This article has been corrected since it first published. The word "Best" has been capitalised in the title and abstract.

\section{Twitter lan C Han @3Y3MD}

Contributors RC, EMS, IH contributed to study design. RC, CRF, DBC, MMC, EHS, EMS, IH contributed to data acquisition. RC, EHS, BAT, RM, IH contributed to interpretation of the data and drafting of the manuscript. All authors contributed to critical review of the work, provided final approval of the version to be published and agree to be accountable for the integrity of the work. IH accepts full responsibility for the work and/or the conduct of the study, had access to the data, and controlled the decision to publish.

Funding Institute for Vision Research, University of lowa, lowa City, lowa; Vitreoretinal Surgery Foundation Research Award, Minneapolis, Minnesota; National Institutes of Health R01-EY024588, P30-EY025580, R01-EY026547.

Competing interests None declared.

Patient consent for publication Not applicable.

Ethics approval This study involves human participants and was approved by University of lowa Institutional Review Board \#200202022 Participants gave informed consent to participate in the study before taking part.

Provenance and peer review Not commissioned; externally peer reviewed.

Data availability statement Data are available upon reasonable request. Not applicable.

Supplemental material This content has been supplied by the author(s). It has not been vetted by BMJ Publishing Group Limited (BMJ) and may not have been peer-reviewed. Any opinions or recommendations discussed are solely those of the author(s) and are not endorsed by BMJ. BMJ disclaims all liability and responsibility arising from any reliance placed on the content. Where the content includes any translated material, BMJ does not warrant the accuracy and reliability of the translations (including but not limited to local regulations, clinical guidelines, terminology, drug names and drug dosages), and is not responsible for any error and/or omissions arising from translation and adaptation or otherwise.

Open access This is an open access article distributed in accordance with the Creative Commons Attribution Non Commercial (CC BY-NC 4.0) license, which permits others to distribute, remix, adapt, build upon this work non-commercially, and license their derivative works on different terms, provided the original work is properly cited, appropriate credit is given, any changes made indicated, and the use is non-commercial. See: http://creativecommons.org/licenses/by-nc/4.0/.

ORCID iD

Ian C Han http://orcid.org/0000-0002-2371-727X

\section{REFERENCES}

1 Petrukhin K, Koisti MJ, Bakall B, et al. Identification of the gene responsible for best macular dystrophy. Nat Genet 1998;19:241-7.

2 Stone EM, Andorf JL, Whitmore SS, et al. Clinically focused molecular investigation of 1000 consecutive families with inherited retinal disease. Ophthalmology 2017;124:1314-31.

3 Blodi CF, Stone EM. Best's vitelliform dystrophy. Ophthalmic Paediatr Genet 1990;11:49-59.

4 Marmorstein AD, Marmorstein LY, Rayborn M, et al. Bestrophin, the product of the Best vitelliform macular dystrophy gene (VMD2), localizes to the basolateral plasma membrane of the retinal pigment epithelium. Proc Natl Acad Sci U S A 2000;97:12758-63.
5 Stone EM, Nichols BE, Streb LM, et al. Genetic linkage of vitelliform macular degeneration (best's disease) to chromosome 11q13. Nat Genet 1992;1:246-50.

6 Mullins RF, Oh KT, Heffron E, et al. Late development of vitelliform lesions and flecks in a patient with best disease: clinicopathologic correlation. Arch Ophthalmol 2005;123:1588-94.

7 Gass JD. A clinicopathologic study of a peculiar foveomacular dystrophy. Trans Am Ophthalmol Soc 1974;72:139-56.

8 Gass JDM. Heredodystrophic disorders affecting the pigment epithelium and retina. In: Stereoscopic atlas of macular diseases: diagnosis and treatment. 2nd ed. Mosby: 303-436.

9 Huang D, Swanson EA, Lin CP, et al. Optical coherence tomography. Science 1991;254:1178-81.

10 Battaglia Parodi M, lacono $\mathrm{P}$, Romano F, et al. Spectral domain optical coherence tomography features in different stages of Best vitelliform macular dystrophy. Retina 2018;38:1041-6.

11 Mohler CW, Fine SL. Long-term evaluation of patients with best's vitelliform dystrophy. Ophthalmology 1981;88:688-92.

12 Wang YT, Tadarati M, Scholl HP, et al. Recurrence of vitelliform lesions associated with temporary vision loss in Best vitelliform macular dystrophy. Retin Cases Brief Rep 2016;10:63-71.

13 Boon CJF, Klevering BJ, Leroy BP, et al. The spectrum of ocular phenotypes caused by mutations in the BEST1 gene. Prog Retin Eye Res 2009;28:187-205.

14 MacDonald IM, Lee T, Lawrence J. Bestrophinopathies. In: Adam MP, Ardinger HH, Pagon RA, et al, eds. Seattle, WA: GeneReviews((R)), 1993.

15 Querques G, Forte R, Querques L, et al. Natural course of adultonset foveomacular vitelliform dystrophy: a spectral-domain optical coherence tomography analysis. Am J Ophthalmol 2011;152:304-13.

16 Querques G, Zerbib J, Santacroce R, et al. Functional and clinical data of Best vitelliform macular dystrophy patients with mutations in the BEST1 gene. Mol Vis 2009;15:2960-72.

17 Wabbels B, Preising MN, Kretschmann U, et al. Genotypephenotype correlation and longitudinal course in ten families with Best vitelliform macular dystrophy. Graefes Arch Clin Exp Ophthalmol 2006;244:1453-66.

18 Parodi MB, Arrigo A, Bandello F. Optical coherence tomography angiography quantitative assessment of macular neovascularization in Best vitelliform macular dystrophy. Invest Ophthalmol Vis Sci 2020;61:61.

19 Roberts MF, Fishman GA, Roberts DK, et al. Retrospective, longitudinal, and cross sectional study of visual acuity impairment in choroideraemia. Br J Ophthalmol 2002;86:658-62.

20 Kay CN, Abramoff MD, Mullins RF, et al. Three-dimensional distribution of the vitelliform lesion, photoreceptors, and retinal pigment epithelium in the macula of patients with Best vitelliform macular dystrophy. Arch Ophthalmol 2012;130:357-64.

21 Kumar V, Chatra K. Fibrotic pillar leads to focal choroidal excavation in Best vitelliform dystrophy. Graefes Arch Clin Exp Ophthalmol 2018;256:2083-7.

22 Shahzad R, Siddiqui MAR. Choroidal neovascularization secondary to Best vitelliform macular dystrophy detected by optical coherence tomography angiography. J Aapos 2017;21:68-70.

23 Battaglia Parodi M, Casalino G, lacono P, et al. The expanding clinical spectrum of choroidal Excavation in macular dystrophies. Retina 2018;38:2030-4.

24 Braimah IZ, Rapole S, Dumpala S, et al. Focal choroidal Excavation in retinal dystrophies. Semin Ophthalmol 2018;33:161-6.

25 Lee JH, Lee WK. Choroidal neovascularization associated with focal choroidal excavation. Am J Ophthalmol 2014;157:710-8.

26 Jampol LM, Shankle J, Schroeder R, et al. Diagnostic and therapeutic challenges. Retina 2006;26:1072-6.

27 Margolis R, Mukkamala SK, Jampol LM, et al. The expanded spectrum of focal choroidal excavation. Arch Ophthalmol 2011:129:1320-5.

28 Coussa RG, Binkley EM, Wilkinson ME, et al. Predominance of hyperopia in autosomal dominant Best vitelliform macular dystrophy. Br J Ophthalmol 2020. doi:10.1136/bjophthalmol-2020-317763. [Epub ahead of print: 26 Nov 2020].

29 Wakabayashi Y, Nishimura A, Higashide T, et al. Unilateral choroidal excavation in the macula detected by spectral-domain optical coherence tomography. Acta Ophthalmol 2010;88:e87-91.

30 Abràmoff MD, Mullins RF, Stone EM. Outer segment length in different best disease genotypes. JAMA Ophthalmol 2014;132:1152-3. 